\title{
Stereoselektive Synthese von C-Glycosiden mit einem Glycosyl-Dianion * *
}

\section{Von Valentin Wittmann und Horst Kessler*}

Die Synthese von C-Glycosiden ${ }^{[1]}$ hat in den letzten Jahren zunehmend an Bedeutung gewonnen. Dies ist zum einen auf die Präsenz C-glycosidischer oder davon abgeleiteter Teilstrukturen in zahlreichen Naturstoffen ${ }^{[2]}$ zurückzuführen, zum anderen sind C-Glycoside potentielle Antimetaboliten $^{[3]}$. Die klassischen Synthesen nutzen - in Analogie zu den O-Glycosidsynthesen - die in 1-aktivierten Zuckern vorgegebene positive Polarisierung des anomeren Zentrums durch den Angriff eines geeigneten C-Nucleophils aus. Auch die Verwendung von Glycosyl-Radikalen ${ }^{[1,4]}$ und Glycosyliden-Carbenen ${ }^{[5]}$ wurde beschrieben. Die direkte 1-C-Lithiierung von Zuckern führt in der Regel zur Eliminierung funktioneller Gruppen in 2-Position ${ }^{[6]}$. Paulsen et al. gelang erstmals die C-Verlängerung offenkettiger Kohlenhydrate durch Umpolung der Reaktivität am anomeren Zentrum über Dianionen von Hydroxy-1,3-dithianen ${ }^{[7]}$. Bei den Pyranosen war das Konzept der Umpolung bisher auf 1-Nitrozucker $1^{[8]}, \quad 2$-Desoxyzucker $2\left(\mathrm{X}=\mathrm{H}^{[6 \mathrm{a}, 9]}, \quad \mathrm{SO}_{2} \mathrm{Ph}^{[10]}\right.$, $\left.{ }^{\oplus} \mathrm{PPh}_{3}{ }^{[11]}, \quad \mathrm{CO}_{2} \mathrm{R}^{[12]}\right)$ und Glycale $3 \quad\left(\mathrm{X}=\mathrm{H}^{[9 \mathrm{~d}, 13]}\right.$, $\mathrm{SOPh}^{[14]}, \mathrm{SPh}^{[15]}, \mathrm{OBn}^{[15]}$ ) beschränkt. Die Nitrozucker 1 führen nach C-Verlängerung und anschließender Substitution der Nitro- durch eine Hydroxygruppe zu höheren Ketopyranosen mit äquatorialer Konfiguration des eingeführten Restes. Die Glycale 3 ermöglichen ein nachträgliches Einführen der fehlenden Sauerstoffunktionalität und bieten einen Zugang zu $\beta$-C-Glycosiden.

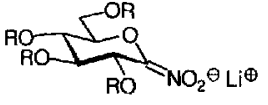

1

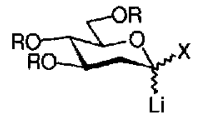

2

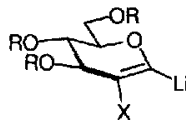

3

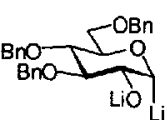

4

Wir möchten hier die Verwendung der Dilithiumverbindung 4 zur C-Glucosidsynthese vorstellen. Die OLi-Gruppe in Position 2 verhindert effizient die $\beta$-Eliminierung. Durch

$\left.{ }^{*}\right]$ Prof. Dr. H. Kessler, Dipl.-Chem. V. Wittmann

Organisch-chemisches Institut der Technischen Universität München Lichtenbergstraßc 4, D-85747 Garching

Telefax: Int. $+89 / 3209-3210$

[**] Diese Arbeit wurde vom Fonds der Chemischen Industrie und von der Deutschen Forschungsgemeinschaft gefördert. V. W. dankt dem Fonds der Chemischen Industrie für ein Doktorandenstipendium. 
Umsetzung mit Elektrophilen gelangt man nunmehr direkt zu 2-Hydroxy-substituierten C-Glucopyranosiden mit definierter $\alpha$-Konfiguration (siehe Schema 1).

Dianionen des allgemeinen Typs 5 lassen sich aus $\beta$ Hydroxyorganoquecksilber-Verbindungen ${ }^{[16]}$, Chlorhydrinen $^{[17]}$, Epoxiden ${ }^{[18]}$, Carbonylverbindungen ${ }^{[10 \mathrm{a}, 19]}$ oder Hydroxy-1,3-dithianen ${ }^{[7]}$ generieren und wurden einerseits als Carbanionen zur C-C-Verknüpfung $(\mathbf{5} \rightarrow \mathbf{6}, \quad$ El. $=$ Elektrophil) ${ }^{[7,16-18,19 a]}$, andererseits zur Olefinsynthese via $\mathrm{Li}_{2} \mathrm{O}$-Eliminierung $(\mathbf{5} \rightarrow \mathbf{7})^{[19,20]}$ eingesetzt.

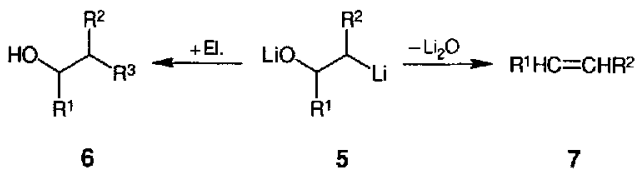

Ob sich 4 zur C-Verlängerung am anomeren Zentrum eignet oder ob die Eliminierung zum Glucal die bevorzugte Reaktion ist, war nicht ohne weiteres abzusehen. So wurde beispielsweise berichtet ${ }^{[17]}$, daß Verbindungen 5 mit Lithium an einem primären C-Atom $\left(R^{2}=H\right)$ bis etwa $-60^{\circ} \mathrm{C}$ stabil sind. Im Fall von $\mathrm{R}^{2}=$ Alkyl dagegen zersetzen sie sich selbst bei $-100^{\circ} \mathrm{C}$ spontan unter $\beta$-Eliminierung zum Olefin. Lediglich Verbindungen 5 mit stabilisierenden Substituenten, wie $\mathrm{R}^{2}=\mathrm{Ph}$ oder $\mathrm{CO}_{2} \mathrm{Et}$, oder das cyclische $\beta$-Lithiocyclohexanolat $\left(\mathrm{R}^{1}, \mathrm{R}^{2}=-\left(\mathrm{CH}_{2}\right)_{4}-\right)$ lassen sich $\mathrm{zu}$ den entsprechenden deuterierten Alkoholen $6\left(R^{3}=D\right)$ umsetzen. Die Arbeiten von Beau und Sinay ${ }^{[10]}$ machen das intermediäre Auftreten des Dilithiumderivats 8 wahrscheinlich. Auch hier ist eine Protonierung ohne $\beta$-Eliminierung möglich.

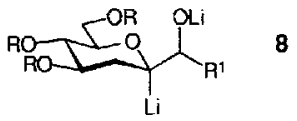

In einem ersten Versuch versetzten wir den in 2-Position ungeschützten Zucker $\mathbf{9}^{[21]}$ in $\mathrm{THF}$ bei $-78^{\circ} \mathrm{C}$ mit 1.1 Äquivalenten n-Butyllithium (zur Erzeugung des Alkoholats) und nach 15 min mit 2.2 Aquivalenten Naphthalinlithium. Nach weiteren 15 min konnte nach Zusatz von MeOD, saurer Aufarbeitung und Kieselgelchromatographie ein Gemisch aus deuteriertem Zucker $10(66 \%)$, der entsprechenden $\beta$-konfigurierten Verbindung $(3.1 \%)$ und der protonierten Verbindung $11(17 \%)$ isoliert werden (Schema 1). Diese Produkte lassen auf das Dianion 4 als Zwischenstufe schließen. Die axiale Stellung des Deuteriums in $\mathbf{1 0}$ ergibt sich aus dem ${ }^{1} \mathrm{H}-\mathrm{NMR}$-Spektrum $\left(250 \mathrm{MHz}, \mathrm{CDCl}_{3}\right.$,
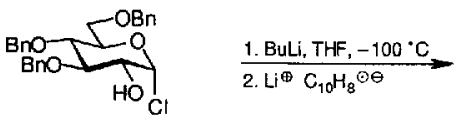

4

9
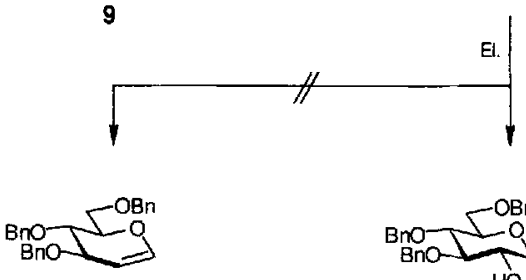

17

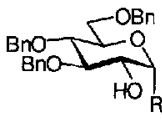

$10-16$

Schema 1. Synthese der $\alpha$-C-Glucoside 10-16 (vgl. Tabelle 1). $\mathrm{Bn}=\mathrm{CH}_{2} \mathrm{Ph}$, El. = Elektrophil.
${ }^{3} J_{\mathbf{H}-1, \mathrm{H}-2}=5.3 \mathrm{~Hz}$ ). Das $\alpha / \beta$-Verhältnis von $21: 1$ wurde durch Deuterium-NMR-Spektroskopie bestimmt. $\mathrm{Li}_{2} \mathrm{O}$-Eliminierung, die zu 3,4,6-Tri- $O$-benzyl-D-glucal 17 führen würde, findet unter diesen Bedingungen offensichtlich nicht statt.

Die höchsten Ausbeuten erhielten wir bei schneller Zugabe der Naphthalinlithium-Lösung. Um dabei ein übermäßiges Ansteigen der Reaktionstemperatur zu vermeiden, sollte die Naphthalinlithium-Lösung vorgekühlt werden, was gewisse Probleme bereitet, da die Löslichkeit von Naphthalinlithium in THF bei $-78^{\circ} \mathrm{C}$ merklich vermindert ist. Einfacher ist es daher, bei $-100^{\circ} \mathrm{C} \mathrm{zu}$ arbeiten und das Reagens ungekühlt zuzuspritzen. Tabelle 1 zeigt die nach dieser Vorgehensweise erhaltenen Ergebnisse.

Tabelle 1. Ergebnisse der C-Glucosidsynthesen nach Schema 1

\begin{tabular}{|c|c|c|c|}
\hline Elektrophil & [rodukt $[\mathrm{a}]$ & $\mathrm{R}$ & Ausb. $[\%][b]$ \\
\hline $\mathrm{MeOD}$ & 10 & $D$ & 75 \\
\hline $\mathrm{MeOH}$ & 11 & $\mathrm{H}$ & 82 \\
\hline $\mathrm{MeCHO}$ & $12 \mathrm{a} / \mathrm{b}$ & $\mathrm{CH}(\mathrm{OH}) \mathrm{Me}$ & 62 \\
\hline $\mathrm{PhCHO}$ & $13 \mathbf{a} / \mathbf{b}$ & $\mathrm{CH}(\mathrm{OH}) \mathrm{Ph}$ & 70 \\
\hline iPrCHO & $14 a / b$ & $\mathrm{CH}(\mathrm{OH}) i \mathrm{Pr}$ & 59 \\
\hline $\mathrm{HCHO}$ & $15[23]$ & $\mathrm{CH}_{2} \mathrm{OH}$ & 17 \\
\hline $\mathrm{MeI}[\mathrm{c}]$ & 16 & $\mathrm{Me}$ & 72 \\
\hline
\end{tabular}

[a] Die Produkte wurden durch ${ }^{1} \mathrm{H}$ - und ${ }^{13} \mathrm{C}-\mathrm{NMR}$-Spektroskopie (siehe Tabellc 2) sowic FAB-MS charakterisiert. [b] Ausheuten an $\alpha$-Anomeren nach Kieselgelchromatographie. [c] Zusatz von $10 \%$ CuI.

Unsere Aufmerksamkeit richtete sich nun auf die Möglichkeit, den lithiierten Zucker zur Synthese von $\alpha$-C-Glucosiden einzusetzen. Die Reaktion von 4 mit Acetaldehyd lieferte die Diastereomerenmischung $12 \mathbf{a} / \mathbf{b}^{[22]}$ (Verhältnis 1.4:1) in $62 \%$ Ausbeute. In gleicher Weise reagierten Benzaldehyd und Isobutyraldehyd zu den Mischungen 13a/b (1:1, $70 \%$ ) bzw. 14a/b $(1: 1,59 \%)$. In allen Fällen entstanden mit hoher Selektivität $(\alpha / \beta \geq 20: 1)$ die axial konfigurierten $\mathrm{C}$ Glucoside. Die Verwendung von gasförmigem Formaldehyd führte nur in einer unbefriedigenden Ausbeute von $17 \%$ zum gewünschten Hydroxymethyl-C-glucosid 15. Statt dessen beobachteten wir die Bildung großer Mengen an Polyoxymethylen. Ohne Probleme dagegen verlief die Um-

Tabelle 2. Ausgewähltc ${ }^{1} \mathrm{H}$ - und ${ }^{13} \mathrm{C}-\mathrm{NMR}$-chemische Verschiebungen $(\delta$ Werte) und Kopplungskonstanten $(J \mathrm{~Hz}])$ von $10-16[a, b]$. ax $=a x i a l$, äq $=$ äquatorial, $\mathrm{n} . \mathrm{b} .=$ nicht bestimmbar.

\begin{tabular}{|c|c|c|c|c|c|c|c|}
\hline Verb. & $\mathrm{H}-1$ & $\mathrm{H}-2$ & $\mathbf{H}-\mathbf{1}^{\prime}$ & C-1 & $\mathrm{C}-2$ & $\mathrm{C}-1^{\prime}$ & ${ }^{3} J_{H-1 . H} 2$ \\
\hline 10 & 3.95 & 3.69 & & 69.1 & 70.0 & & 5.3 \\
\hline 11 & $\begin{array}{l}3.97(a ̈ q) \\
3.18(a x)\end{array}$ & 3.69 & & 69.5 & 70.1 & & $\begin{array}{r}5.3(\mathrm{aq}-\mathrm{ax}) \\
11.1(\mathrm{ax}-\mathrm{ax})\end{array}$ \\
\hline $12 a$ & 3.50 & 3.69 & 4.04 & 73.6 & 67.6 & 67.7 & 1.6 \\
\hline $12 b$ & 3.57 & 3.98 & 4.10 & 73.7 & 68.4 & 67.0 & 2.9 \\
\hline $13 a$ & 3.75 & 3.34 & 4.97 & 73.8 & 66.1 & 73.9 & 1.0 \\
\hline $13 \mathrm{~b}$ & 3.93 & 3.93 & 5.00 & 72.4 & 67.4 & 74.0 & n.b. $[\mathrm{c}]$ \\
\hline $14 a$ & 3.78 & 3.75 & 3.60 & 69.2 & 68.9 & 76.8 & $<1.0$ \\
\hline $14 b$ & 3.77 & 3.97 & 3.77 & 69.8 & 68.1 & 74.3 & 2.0 \\
\hline 15 & 3.97 & 3.76 & $\begin{array}{l}3.76 \\
3.87\end{array}$ & 71.1 & 68.7 & 62.2 & 2.7 \\
\hline 16 & 4.09 & 3.61 & 1.23 & 68.1 & 70.4 & 14.2 & 3.8 \\
\hline
\end{tabular}

[a] NMR-Spektren in $\mathrm{CDCl}_{3}$ bei $300 \mathrm{~K}$, interner Standard TMS. ${ }^{\mathrm{I}} \mathrm{H}$ $500 \mathrm{MHz},{ }^{13} \mathrm{C}: 125 \mathrm{MHz}$. [b] Zur besseren Vergleichbarkcit wurden die Atome wie im Eduktzucker 9 numeriert:

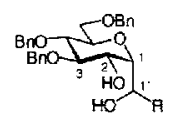

[c] Gleiche chemische Verschiebung von $\mathrm{H}-1$ und $\mathrm{H}-2$. 
setzung des Dianions 4 mit MeI, die in $72 \%$ das $\alpha$-Methyl-Cglucosid 16 lieferte. Bei dieser Alkylierung erwies sich der Zusatz von $10 \% \mathrm{CuI}$ vor Zugabe des Elektrophils als sinnvoll (Ausbeute ohne CuI: $58 \%$ ).

Bemerkenswert erscheint uns, daß wir bei keiner Reaktion die Bildung von Tribenzylglucal 17 beobachten konnten. Somit erweist sich die Glucosyllithiumverbindung 4 als eine bei $-78^{\circ} \mathrm{C}$ stabile Spezies, die sich mit Elektrophilen unter Retention der Konfiguration am anomeren Zentrum zu einer Vielzahl an 2-Hydroxy- $\alpha$-C-Glucosiden umsetzen läßt. Der zu ihrer Erzeugung benötigte Zucker 9 ist eine kristalline, bei $-20^{\circ} \mathrm{C}$ lagerbare Substanz ${ }^{[21]}$ und aus Acetobromglucose in vier Schritten mit einer Gesamtausbeute von etwa $55 \%$ bequem erhältlich.

\section{Arbeitsvorschrift}

Zu einer $0.15 \mathrm{~m}$ Lösung von 9 in trockenem THF werden unter Schutzgas bei $-100{ }^{\circ} \mathrm{C} 1.1$ Äquiy. $n \mathrm{BuLi}(1.6 \mathrm{M}$ in Hexan) und nach einigen Minuten zügig 2.2 Äquiv. Naphthalinlithium ( $1 \mathrm{M}$ in THF) zugetropft. Nach $15 \mathrm{~min}$ gibt man 1.5 Äquiv. Aldehyd oder MeI zu, rührt $1 \mathrm{~h}$ bei $-100^{\circ} \mathrm{C}$ und läßt auf Raumtemperatur erwärmen. Zur Aufarbeitung wird die Reaktionsmischusg mit gesüttigter wäßriger $\mathrm{NH}_{4} \mathrm{Cl}$-Lösung versetzt und mit Dichlormethan extrahiert. Nach Trocknen der organischen Phase über $\mathrm{MgSO}_{4}$ wird das Produkt an Kieselgel chromatographiert (Eluent: Toluol/Ethylacetat oder Hexan/Ethylacetat).

Eingegangen am 8. Februar 1993 [Z 5856]
[18] a) T. Cohen, I.-H. Jeong, B. Mudryk, M. Bhupathy, M. M. A. Awad, d. Org. Chem. 1990, 55, 1528-1536; b) E. Bartmann, Angew. Chem. 1986 98, 629-631; Angew. Chem. Int. Ed. Engl. 1986, 25, 653-654.

[19] a) J. Barluenga, J. L. Fernandez-Simon. J. M. Concellon, M. Yus, J. Chem. Soc. Perkin Trans. 1 1988, 3339-3343; b) J. Barluenga, F. Alvarez, J. M. Concellon, M. Yus, J. Chem. Res. (S) 1987, 402-403; (M) 3265-3285.

[20] K. N. Gurudutt, B. Ravindranath, Tetrahedron Lett. 1980, 21, 1173-1174.

[21] H. Yamaguchi, C. Schuerch, Carbohdr. Res. 1980, 81, 192-195.

[22] Die Kennzeichnungen a und $\mathbf{b}$ beziehen sich auf die durch Addition an beide stereoheterotopen Seiten des prochiralen Aldehyds entstehenden Diastereomere, die sich durch Kieselgelchromatographie trennen lassen.

[23] G. Stork, H. S. Suh, G. Kim, I. Am. Chem. Soc. 1991, 113, 70547056.

[1] Eine ausgezeichnete Übersicht giht: M. H. D. Postema, Tetrahedron 1992. $48,8545-8599$. Siehe auch die dort zitierte Literatur.

[2] a) G. Franz, M. Grün, Planta Med. 1983, 47,131-140; b) S. Hanessian, Total Synthesis of Natural Products: The ,Chiron' Approach, Pergamon Press, Oxford, 1983.

[3] a) C. Bertozzi, M. Bednarski, Carbohydr. Res. 1992, 223, 243-253; b) K. Krohn, H. Heins, K. Wielckens, J. Med. Chem. 1992, 35, 511-517 c) R. R. Schmidt, H. Dietrich, Angew. Chem, 1991, 103, 1348-1349; Angew. Chem. Int Ed. Engl. 1991, 30, 1328-1329.

[4] a) B. Giese, T. Linker, R. Muhn, Tetrahedron 1989, 45, 93.5-940; b) $\mathrm{H}$ Kessler, V. Wittmann, M. Köck, M. Kottenhahn, Angew. Chem. 1992, 104 , 874-877; Angew. Chem. Int. Ed. Engl. 1992, 31, 902-904.

[5] A. Vasella, C. Witzig, R. Husi, Helv. Chim. Acta 1991, 74, 1362-1372.

[6] a) J-M. Lancelin, L. Morin-Allory, P. Sinaÿ, J. Chem. Soc. Chem. Commun. 1984, 355-356; b) R. R. Schmidt, J. Kast, Tetrahedron Lett. 1986, 27 , $4007-4010$.

[7] H. Paulsen, K. Roden, V. Sinnwell, P. Luger, Liebigs Ann. Chem. 1981, $2009-2027$.

[8] a) B. Aebischer, J. H. Bieri. R. Prewo, A. Vasella, Hetv. Chim. Acra 1982 , 65, 2251-2272; b) K. Mahmood, A. Vasella, B. Bernet, ibid. 1991, 74, $1555-1583$.

[9] a) P. Lesimple, J.-M. Bcau, P. Sinaÿ, J. Chem. Soc. Chem. Commun. 1985 , 894-895; b) J.-M. Beau, P. Sinaÿ, Tetrahedron Lett. 1985, 26,6185-6188; c) P. Lesimple, J-M. Beau, P. Sinaÿ, Carbohydr. Res. 1987, 171, 289-300; d) J, Prandi, C. Audin, J.-M. Beau, Tetrahedron Lett. 1991, 32, 769-772 e) D. K. Hutchinson, P. L. Fuchs, J. Am. Chem. Soc. 1987, 109, 49304939 .

[10] a) J.-M. Beau, P. Sinaÿ, Tetrahedron Lett. 1985, 26,6189-6192; b) J.-M Beau, P. Sinaÿ. ibid. 1985, 26, 6193-6196.

[11] J. B. Ousset, C. Mioskowski, Y.-L. Yang, J. R. Falck, Tetrahedron Lett. 1984, 25, 5903-5906; vgl. auch S. V. Ley, B. Lygo, ibid. 1984, 25, 113-116.

[12] a) D. Crich, L. B. L. Lim, Tetrahedron Lett. 1999, 31, 1897-1900; b) K. Wallimann, A. Vasella, Helv. Chim. Acta 1991, 74, 1520-1532.

[13] a) K. C. Nicolaou, C.-K. Hwang, M. E. Duggan, J. Chem. Soc. Chem. Commun. 1986. 925-926; b) S. Hanessian, M. Martin, R. C. Desai, ibid. 1986, $926-927$; c) P. Lesimple, J.-M. Beau, G. Jaurand, P. Sinaȳ, Tetrahedron Lett. 1986, 27, 6201-6204; d) K. A. Parker, C. A. Coburn, J. Am. Chem. Soc. 1991, 113, 8516-8518.

[14] a) R. Preuss, R. R. Schmidt, Liebigs Ann. Chem. 1989, 429-434; b) R. R. Schinidt, R. Preuss, Tetrahedron Lett. 1989, 30,3409-3412; c) S. Maier, R. Preuss, R. R. Schmidt, Liebigs Ann. Chem. 1990, 483-489.

[15] R. R. Schmidt, R. Preuss, R. Betz, Tetrahedron Lett. 1987, 28, 6591- 6594.

[16] J. Barluenga, F. J. Fananas, J. Villamana, M. Yus, J. Org. Chem. 1982, 47, $1560 \cdots 1564$.

[17] a) C. Najera, M. Yus, D. Seebach, Helv. Chim. Acta 1984, 67, 289-300; b) J. Barluenga, J. Florez, M. Yus, J. Chem. Soc. Perkin Trans. 1 1983, 3019-3026. 